\title{
Article
}

\section{Three-dimensional Viscoelastic Analysis of Multi-Layer Polymer Flow by Numerical Simulation}

\author{
Takeshi INOMOTO*,** and Toshihisa KAJIWARA $^{* *}$ \\ "Engineering Development Center, Toray Industries, Inc. \\ 3-3-6 Sonoyama, Otsu 520-0842, Japan \\ ** Department of Chemical Engineering, Faculty of Engineering, Kyushu University \\ 744 Motooka, Nishi-ku, Fukuoka 819-0395, Japan \\ (Received : November 4, 2008)
}

\begin{abstract}
We performed three-dimensional viscoelastic analysis of multi-layer polymer flow by numerical simulation, and investigated the effects of the elongational properties on the encapsulation phenomenon in the coextrusion process. The K-BKZ model with multiple relaxation times was used as viscoelastic constitutive equation, and the PSM type damping function was used for this model. This model is convenient to investigate the effects of the elongational properties, because the parameter $\beta$ in this model can control only the elongational properties. Also we can extract the effects only of the elongational properties, because the second normal stress difference in this model is zero. In this study, we assumed that multi-layer polymer flow of Fluid I (less viscous) and Fluid II (more viscous) flows in three kinds of channels with different taper angle (parallel, tapered contraction and tapered expansion). As a result, by increasing the $\beta$ ratio between Fluid I and Fluid II $\left(\beta_{I} / \beta_{I I}\right)$ from 0.1 to 10.0 , the degree of encapsulation (DE) hardly changed at confluence, increased at contraction part and decreased at expansion part. These results mean that the elongational properties clearly influence the encapsulation phenomenon especially at contraction part and expansion part.

Key Words: Encapsulation phenomenon / Three dimensional viscoelastic analysis / Finite element method / Elongational properties
\end{abstract}

\section{INTRODUCTION}

In the coextrusion process, polymer melts with different properties are extruded from two or more extruders and form a multilayered system by merging in a die (called the multimanifold method) or in a feedblock (called the feedblock method). It is very important to keep the uniformity of the layer thickness in this process. However, there are some problems such as the encapsulation phenomenon in which the less viscous fluid encloses the more viscous fluid and interfacial instability at an irregular interface occurs between melts. Then it is very difficult to keep the uniformity of the layer thickness.

Viscosity and elasticity stratification are known to cause interfacial instabilities that develop in the primary flow direction, and these have received much experimental and theoretical investigation. ${ }^{1,2,3)}$ On the other hand, the encapsulation phenomenon involves distortion of the interface in the plane orthogonal to the primary flow, i.e., the cross section.

A major factor in the encapsulation phenomenon has been identified as the viscosity difference between the two continuous phases. In viscous encapsulation, the less viscous fluid encloses the more viscous fluid in pipe flows. ${ }^{4,5)}$ The viscosity contrast determines both the direction and the degree of encapsulation. ${ }^{4)}$ A “minimum viscous dissipation principle” has been invoked to explain the interface shape found in experiment, ${ }^{6,7)}$ although such a variational principle is not rigorously justifiable for flows with interfaces, and is known to fail under certain conditions. ${ }^{8)}$ Everage ${ }^{9)}$ carried out both experimental and theoretical studies on the transient interface motion in a bicomponent tube flow of a nylon/nylon system, and identified two stages in viscous encapsulation.

Another factor, specific to viscoelastic fluids, is the elastic normal stresses. When viscoelastic fluids are sheared in a pipe flow, the second normal stress difference N2 amounts to a lateral forcing in the plane of the cross section. This may cause interfacial motion through two mechanisms. First, if the cross section is noncircular, secondary flows will arise even within a single-component homogeneous fluid. This is a well-known effect and a summary of the literature can be found in Yue et al. ${ }^{10)}$. This geometric effect will distort the interface between 
two polymers regardless of their rheological differences. ${ }^{11)}$ Second, "elastic stratification" in terms of differing N2 between the components will produce an imbalance of force and thus interfacial deformation even in a circular pipe. White et al. ${ }^{12)}$, through a simple theoretical analysis, showed that the component with a higher second normal stress coefficient tends to protrude into the other one, and eventually be encapsulated. In bicomponent coextrusion through noncircular dies ${ }^{2,13}$, both mechanisms are at work. For brevity, we will use the term elastic encapsulation to refer to interfacial motion driven by elastic normal stresses, even though the secondary flow in noncircular channels usually does not lead to complete encirclement of one component by the other. ${ }^{11)}$

Thus a lot of researchers have investigated the effect of the shear properties (especially the second normal stress difference) on the encapsulation phenomenon. But there is little report on the effect of the elongational properties on this phenomenon. So, in this study, we performed threedimensional viscoelastic analysis of multi-layer polymer flow by numerical simulation, and investigated the effects of the elongational properties on the encapsulation phenomenon in the coextrusion process.

\section{GOVERNING EQUATION}

In this study, we assumed that the fluid is incompressible and flow is steady creeping flow under isothermal condition. The conservation equations of mass and momentum are represented as follows:

$$
\begin{aligned}
& \nabla \cdot \boldsymbol{v}_{k}=0, k=I, I I \\
& -\nabla p_{k}+\nabla \cdot \boldsymbol{\tau}_{k}=0, k=I, I I
\end{aligned}
$$

where $\boldsymbol{v}$ is the velocity vector, $p$ is the hydrostatic pressure.

The K-BKZ model with multiple relaxation times was used as viscoelastic constitutive equation, and the PSM type damping function ${ }^{14)}$ was used for this model. These models are as follows:

$$
\begin{aligned}
& \boldsymbol{\tau}=\sum_{i=1}^{N} \int_{-\infty}^{t} \frac{G_{i}}{\lambda_{i}} \exp \left(-\frac{t-t^{\prime}}{\lambda_{i}}\right) h\left(\boldsymbol{C}_{t}^{-1}\left(t^{\prime}\right)\right) d t^{\prime} \quad(\mathrm{K}-\mathrm{BKZ}) \\
& \text { PSM type } h=\frac{\alpha}{\alpha+\beta \mathbf{I}_{C^{-1}}+(1-\beta) \mathbf{I}_{C}+3}
\end{aligned}
$$

where $\lambda_{i}$ and $G_{i}$ are the relaxation time and the relaxation modulus coefficient in the $i$-th relaxation mode. $\alpha$ and $\beta$ are the non-linear parameters under the large deformations, $\boldsymbol{C}$ and $\boldsymbol{C}^{-1}$ are Finger and Cauchy strain tensor and $\mathbf{I}_{C}$ and $\mathbf{I}_{C}{ }^{-1}$ are the invariant of $\boldsymbol{C}$ and $\boldsymbol{C}^{-1}$ respectively. Here, the parameter $\beta$ can control only the elongational properties.

\section{ANALYSES TECHNIQUE}

We used the decoupled method for the calculation of the velocity, pressure and stress in order to reduce the matrix size and time required for the computation. First, the velocity and pressure are calculated from Eqs. (1) and (2) for a given stress field. Then the stress is calculated from viscoelastic constitutive equation by using calculated value of velocity. The velocity and pressure are calculated by means of finite element method and the stress is calculated by the streamline integration method. ${ }^{15)}$ The calculated stress is used for the next integration step and the iteration is continued to obtain the converged solution. To increase the numerical stability, we modified the momentum equation by adding the reference viscous stress term to both side of this equation as follows ${ }^{16,17)}$ :

$$
-\nabla p+2 \eta_{\mathrm{r}} \nabla \cdot \boldsymbol{D}^{\text {new }}=-\nabla \cdot \boldsymbol{\tau}+2 \eta_{\mathrm{r}} \nabla \cdot \boldsymbol{D}^{\text {old }}
$$

where $\eta_{\mathrm{r}}$ is reference viscosity, and "old" and "new" mean the previous known and next unknown values, respectively. There is suitable range of $\eta_{\mathrm{r}}$ because too small value shows less effect of stability, whereas too larger value leads to noticeable error. So we determined $\eta_{\mathrm{r}}$ by Von Meses' method as follows ${ }^{17)}$ :

$$
\eta_{r}=\frac{\hat{\boldsymbol{\tau}}}{\hat{\boldsymbol{D}}}
$$

where $\hat{\boldsymbol{\tau}}$ is Von Mises' equivalent stress and $\hat{\boldsymbol{D}}$ is the equivalent strain rate, respectively. These are represented as

$$
\begin{aligned}
& \hat{\boldsymbol{\tau}}=\left(\mathbf{I}_{\boldsymbol{\tau}}^{2}-3 \mathbf{I I}_{\boldsymbol{\tau}}\right)^{1 / 2} \\
& \hat{\boldsymbol{D}}=\frac{2}{\sqrt{3}}\left(\mathbf{I I}_{\boldsymbol{D}}\right)^{1 / 2}
\end{aligned}
$$

where $\mathbf{I}_{\boldsymbol{\tau}}$ and $\mathbf{I I}_{\boldsymbol{\tau}}$ are the first and second invariants of the stress tensor and $\mathbf{I I}_{\boldsymbol{D}}$ is the second invariant of the strain rate tensor.

\section{CHANNEL GEOMETRY AND BOUNDARY CONDITIONS}

Fig. 1 shows the channel geometry and the boundary conditions. In this study, we used three kinds of channels with different taper angle $\phi$ (parallel, tapered contraction and tapered expansion). The taper angle $\phi$ in each channels are $0.0 \mathrm{deg}$ (parallel), $45.0 \mathrm{deg}$ (tapered contraction) and -45.0 deg 
(tapered expansion).

We performed the half analysis considering the symmetry of the flow region. We imposed no-slip condition on the wall $(\mathrm{z} / L=0.5)$ : Eq. (9), fully developed flow condition on the inlet $(x / L=-5)$ and outlet $(x / L=16)$ : Eq. (10) and symmetry condition for the symmetry plane $z / L=0$ : Eq. (11).

$$
\begin{aligned}
& v_{k}=0, k=I, I I \\
& \frac{\partial v_{k}}{\partial x}=\frac{\partial w_{k}}{\partial x}=0, k=I, I I \\
& w_{k}=0, \frac{\partial u_{k}}{\partial z}=\frac{\partial v_{k}}{\partial z}=0, k=I, I I
\end{aligned}
$$

where $x, y$, and $z$ are the coordinate directions and $u, v$, and $w$ are the velocity components in $x, y$, and $z$ directions, respectively.

Besides the above conditions, we imposed kinematic conditions Eqs. (12), (13) and (14), and dynamic conditions Eqs. (15), (16) and (17) on the interface for the multilayer flow:

$$
\begin{aligned}
& \boldsymbol{n} \cdot \boldsymbol{v}_{I}=\boldsymbol{n} \cdot \boldsymbol{v}_{I I}=0 \\
& \boldsymbol{t}_{1} \cdot \boldsymbol{v}_{I}=\boldsymbol{t}_{1} \cdot \boldsymbol{v}_{I I} \\
& \boldsymbol{t}_{2} \cdot \boldsymbol{v}_{I}=\boldsymbol{t}_{2} \cdot \boldsymbol{v}_{I I} \\
& \boldsymbol{n} \cdot \boldsymbol{\sigma}_{I}=\boldsymbol{n} \cdot \boldsymbol{\sigma}_{I I} \\
& \boldsymbol{t}_{1} \cdot \boldsymbol{\sigma}_{I}=\boldsymbol{t}_{1} \cdot \boldsymbol{\sigma}_{I I} \\
& \boldsymbol{t}_{2} \cdot \boldsymbol{\sigma}_{I}=\boldsymbol{t}_{2} \cdot \boldsymbol{\sigma}_{I I}
\end{aligned}
$$

where $\boldsymbol{\sigma}$ is the total stress tensor, $\boldsymbol{n}$ is the outward normal vector to the interface and $\boldsymbol{t}_{1}$ and $\boldsymbol{t}_{2}$ are the tangential vectors at the interface.

\section{DETERMINATION OF THE INTERFACE}

The problem is complicated because there exists a fluid/ fluid interface whose position is not known a priori and thus an interface-updating algorithm must be included in the calculation by Picard-type iteration. Pathline method is used to update the interface position. ${ }^{18,19,20)}$ The interface is described by the path of particles traveling downstream from the end of the separating plate because particles at the interface remain in a tangential direction to the interface. Their paths are determined by the following relationship:

$$
\frac{u}{d x}=\frac{v}{d y}=\frac{w}{d z}
$$

and the interface is calculated separately from the calculation of velocity field based on the latest information of converged velocity as follows:

$$
\Delta y=\int \frac{v}{u} d x
$$

where $x, y$, and $z$ are the coordinate directions and $u, v$, and $w$ are the velocity components in $x, y$, and $z$ directions, respectively. The path line method is a simple method but gives slow convergence, and thus the procedure generally requires a large number of iterations to achieve a specified convergence.

After updating the interface position, it is necessary to relocate the inner coordinates of the flow domain of interest. The flow domain is remeshed only in the thickness direction using the predetermined ratio. The inner nodes are located on a set of straight spines; their distance to the upper wall is based on a proportionality factor that is constant throughout the deformation of the mesh. The coordinates of the inner nodes are thus related to the displacement of the interface nodes.

\section{DETERMINATION OF THE CONTACT LINE}

The interface shape was determined by rearranging onto the streamlines obtained from the velocity field. However as explained above, we imposed a no-slip condition on the wall. Because of this boundary condition, the interface on the wall (boundary of fluid/fluid/solid) never moved. A treatment of the contact line is use a contact angle condition, but a very fine mesh of molecular size $\mathrm{e}^{22,23)}$ will be needed to calculate the strict location of the contact line. Therefore, we used the quadratic interpolation for extrapolating the contact line, which was used in previous studies. ${ }^{19,20,21)}$ A concrete treatment of the contact line in our simulation is presented in Fig. 2.

Fig.1. The channel geometry and the boundary condition. 
This technique is the simplest method to calculate the location of the contact line, but it does not consider wall effects and is influenced easily from the mesh. But we thought that these problems are not so important. Because we investigated qualitatively the effects on the encapsulation phenomenon and also the change in interfacial shape was comparatively small.

\section{MATERIAL CONSTANTS}

In this study, we used two kinds of fluid with different viscosity (Fluid I and Fluid II ). The relaxation spectrum and the non-linear parameters of these fluids were determined referring to HDPE data. Table I shows the determined relaxation spectrum. Fig. 3 shows the steady shear viscosity and the first normal stress difference for the K-BKZ model. Fig.4 shows the steady uniaxial elongational viscosity for the K-BKZ model. The parameter $\beta$ can control the elongational properties independent from the shear properties. To investigate the effects of the elongational properties, $\beta_{I}$ is fixed to 0.1 and $\beta_{I I}$ is set to $1.0,0.1$ and 0.01 , i.e., the $\beta$ ratio $\left(\beta_{I} / \beta_{I I}\right)$ is set to $0.1,1.0$ and 10.0 .

Here, by a lot of past reports, we know that the second normal stress difference influence the encapsulation phenomenon significantly. But we can extract the effects only of the elongational properties, because the second normal stress difference is zero in this model.

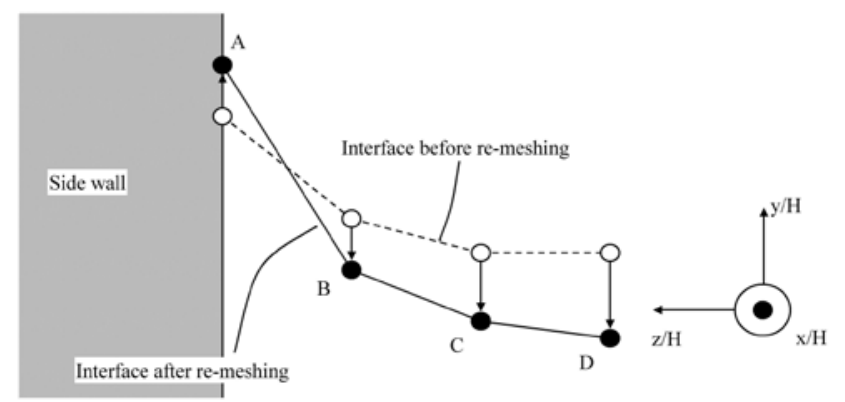

- A is approximated by quadratic interpolation with $\mathrm{B}, \mathrm{C}$ and $\mathrm{D}$

Fig.2. A treatment for the contact line in this study.

Table I. The relaxation spectrum and the non-linear parameters for the K-BKZ model.

\begin{tabular}{|c|c|c|c|c|c|c|c|}
\hline \multicolumn{2}{|c|}{$\mathrm{i}$} & 1 & 2 & 3 & 4 & 5 & 6 \\
\hline \multicolumn{2}{|c|}{$\lambda_{i}$} & $1.00 \times 10^{-3}$ & $1.00 \times 10^{-2}$ & $1.00 \times 10^{-1}$ & $1.00 \times 10^{0}$ & $1.00 \times 10^{1}$ & $1.00 \times 10^{2}$ \\
\hline \multirow{2}{*}{$G_{i}$} & Fluid I & $2.30 \times 10^{5}$ & $9.56 \times 10^{4}$ & $4.15 \times 10^{4}$ & $1.34 \times 10^{4}$ & $3.45 \times 10^{3}$ & $9.67 \times 10^{2}$ \\
\cline { 2 - 7 } & Fluid II & $4.60 \times 10^{5}$ & $1.91 \times 10^{5}$ & $8.30 \times 10^{4}$ & $2.68 \times 10^{4}$ & $6.90 \times 10^{3}$ & $19.3 \times 10^{3}$ \\
\hline
\end{tabular}

Fluid I: $\alpha=7.0, \beta=0.1$

Fluid II: $\alpha=7.0, \beta=1.0,0.1,0.01$

\section{RESULTS AND DISCUSSION}

As a simple criterion for the encapsulation phenomenon, the degree of encapsulation (DE) is defined as

$$
D E=\frac{y_{w}-y_{c}}{L} \times 100(\%)
$$

where $y_{w}$ is the height of the interface at side wall $(\mathrm{z} / \mathrm{L}=0.5)$, $y_{c}$ is the height of the interface at symmetric plane $(z / L=0)$, and $L$ is the characteristic length. In viscous encapsulation, the less viscous fluid (Fluid I ) encloses the more viscous fluid (Fluid II ) and DE is positive in parallel channel.

Fig. 5 shows the change of DE in parallel channel by increasing $\beta_{I} / \beta_{I I}$ from 0.1 to 10.0. In $\beta_{I} / \beta_{I I}=0.1$, DE slightly decreases just after the confluence, rapidly increases and gradually steadies. By increasing $\beta_{I} / \beta_{I I}$, DE hardly changes. This result means that the elongational properties hardly influence the encapsulation phenomenon at confluence.

Fig. 6 shows the change of $\mathrm{DE}$ in tapered contraction channel by increasing $\beta_{I} / \beta_{I I}$ from 0.1 to 10.0 . In any $\beta_{I} / \beta_{I I}$,

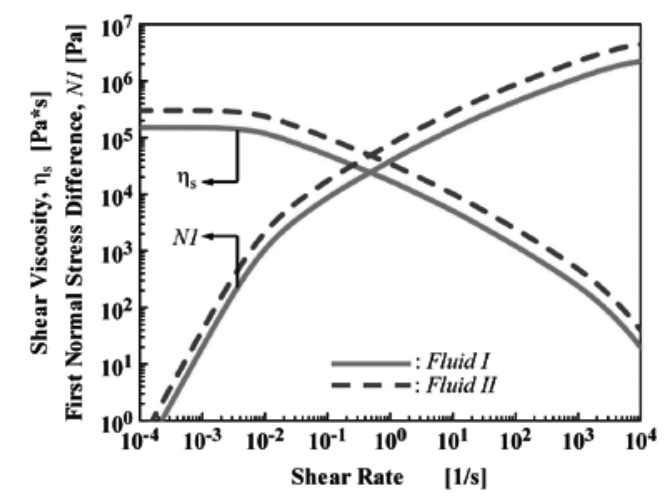

Fig.3. The steady shear viscosity and the first normal stress difference for the K-BKZ model.

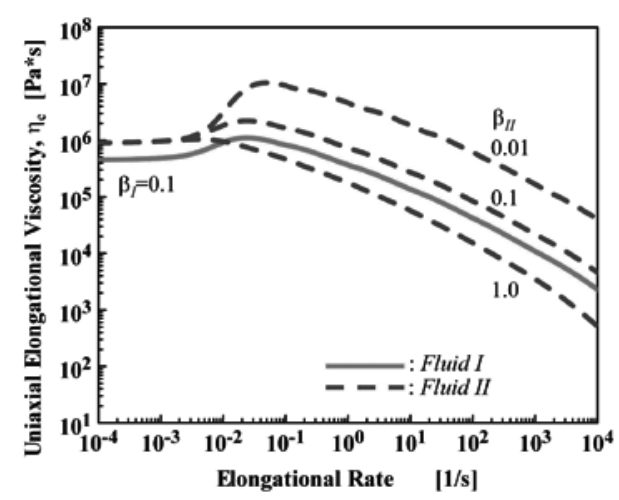

Fig.4. The steady uniaxial elongational viscosity for the K-BKZ model. 
the change of DE just after the confluence is similar to it in parallel channel. In $\beta_{I} / \beta_{I I}=0.1$, DE increases before contraction part, decreases on contraction part and increases after the contraction part. By increasing $\beta_{I} / \beta_{I I}$ from 0.1 to 1.0 , $\mathrm{DE}$ increases more before contraction part. But, by increasing $\beta_{I} / \beta_{I I}$ from 1.0 to 10.0 , DE hardly changes. At contraction part, large elongational rate is generated in the whole of fluid, and one fluid with less elongational viscosity stretches and thins more than the other fluid. Here, by increasing $\beta_{I} / \beta_{I I}$ from 0.1 to 1.0 , the elongational viscosity of Fluid I becomes larger than the one of Fluid II. Thus the interface moves to Fluid I and DE increases. This result means that the elongational properties clearly influence the encapsulation phenomenon at contraction part.

Fig.7 shows the change of DE in tapered expansion channel by increasing $\beta_{I} / \beta_{I I}$ from 0.1 to 10.0 . In any $\beta_{I} / \beta_{I I}$, the change of DE just after the confluence is similar to it in parallel channel. In $\beta_{I} / \beta_{I I}=0.1$, DE decreases before expansion part, increases on expansion part and decreases after the expansion part. By increasing $\beta_{I} / \beta_{I I}$ from 0.1 to 1.0,

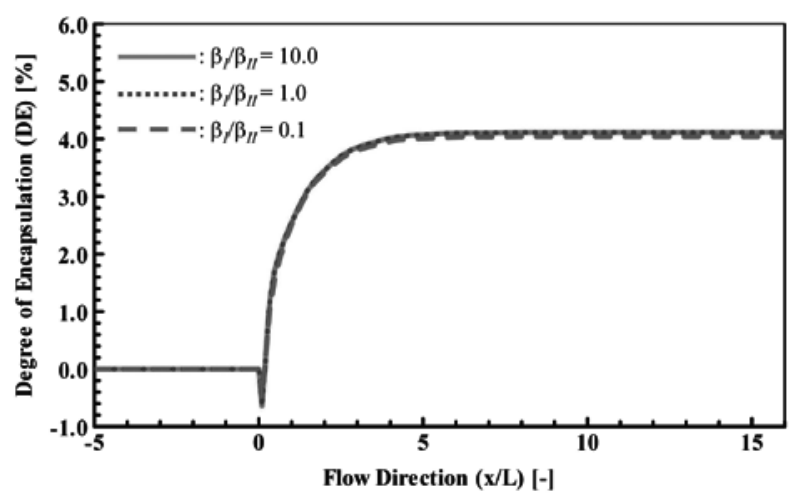

Fig.5. Degree of Encapsulation along flow direction $(\mathrm{x} / \mathrm{L})$ in parallel channel.

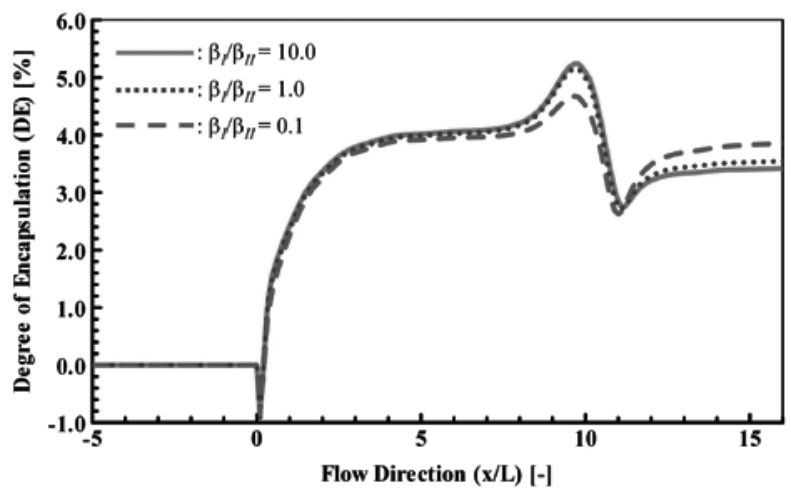

Fig.6. Degree of the Encapsulation along flow direction $(\mathrm{x} / \mathrm{L})$ in tapered contraction channel.
DE decreases more before expansion part. But, by increasing $\beta_{I} / \beta_{I I}$ from 1.0 to 10.0 , DE hardly changes. At expansion part, large negative elongational rate is generated in the whole of fluid, and one fluid with more elongational viscosity shrinks and thickens more than the other fluid. Here, by increasing $\beta_{I} / \beta_{I I}$ from 0.1 to 1.0 , the elongational viscosity of Fluid I becomes larger than the one of Fluid II. Thus the interface moves to Fluid I and DE increases. This result means that the elongational properties clearly influence the encapsulation phenomenon at expansion part, too.

\section{CONCLUSIONS}

We performed three-dimensional viscoelastic analyses of multi-layer polymer flow of Fluid I (less viscous) and Fluid II (more viscous) flows in three kinds of channels with different taper angle (parallel, tapered contraction and tapered expansion) by numerical simulation using the $\mathrm{K}-\mathrm{BKZ}$ model with multiple relaxation times as viscoelastic constitutive equation, and investigated the effects of the elongational properties on the encapsulation phenomenon in the coextrusion process. As a result, by increasing the $\beta$ ratio between Fluid I and Fluid II $\left(\beta_{I} / \beta_{I I}\right)$ from 0.1 to 10.0, the degree of encapsulation (DE) hardly changed at confluence, increased at contraction part and decreased at expansion part. These results mean that the elongational properties clearly influence the encapsulation phenomenon especially at contraction part and expansion part.

In this study, we could show clearly these effects of the elongational on the encapsulation phenomenon. In such small number of layers, these effects of the elongational properties on the encapsulation phenomenon are smaller than the effects of the shear properties (especially the second normal stress

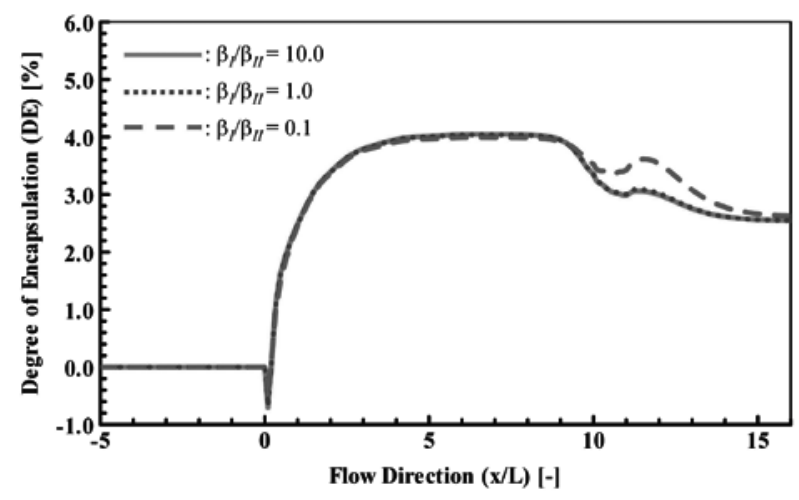

Fig.7. Degree of Encapsulation along flow direction $(\mathrm{x} / \mathrm{L})$ in tapered expansion channel. 
difference). But, in large number of layers, these effects of the elongational properties will be more important. Thus we are going to investigate the effects of the elongational properties on the encapsulation phenomenon in large number of layers.

\section{REFERENCES}

1) Wilson GM, Khomami B, J Rheol, 37 (2), 315 (1993).

2) Khomami B, Ranjbaran MM, Rheol Acta, 36, 345 (1997).

3) Khomami B, Su KC, J Non-Newtonian Fluid Mech, 91, 59 (2000).

4) Lee BL, White JL, Trans Soc Rheol, 18 (3), 467 (1974).

5) Southern JH, Ballman RL, J Polym Sci, 13, 863 (1975).

6) MacLean DL, Trans Soc Rheol, 17 (3), 385 (1973).

7) Elemans PHM, Janssen JMH, Meijer HEH, J Rheol, 34, 1311 (1990).

8) Joseph DD, Renardy YY, Springer-Verlag, New York (1993).

9) Everage AE, Trans Soc Rheol, 19 (4), 509 (1975).

10) Yue P, Dooley J, Feng JJ, J Rheol, 52, 315 (2008).

11) Dooley J, Hyun KS, Hughes K, Polym Eng Sci, 38 (7), 1060 (1998).
12) White JL, Ufford RC, Dharod KR, Price RL, J Appl Polym Sci, 16, 1313 (1972).

13) Dooley J, Rudolph L, J Plast Film Sheeting, 19 (2), 111 (2003).

14) Papanastasiou AC, Scriven LE, Macosko CW, J Rheol, 27, 387 (1983).

15) Luo X-L, Tanner RI, J Non-Newtonian Fluid Mech, 22, 61 (1986).

16) Tanoue S, Kajiwara T, Iemoto Y, Funatsu K, Polymer Eng Sci, 38 (3), 409 (1998).

17) Otsuki Y, Kajiwara T, Funatsu K, Polym Eng Sci, 37, 1171 (1997).

18) Mavridis H, Hrymak AN, Vlachopoulos J, AIChE J, 33, 410 (1987).

19) Karagiannis A, Hrymak AN, Vlachopoulos J, Rheol Acta, 29, 71 (1990).

20) Gifford WA, Polym Eng Sci, 37, 315 (1997).

21) Torres A, Hrymak AN, Vlachopoulos J, Rheol Acta, 32, 513 (1993).

22) Lowndes J, J Fluid Mech, 101, 631 (1980).

23) Bazhlekov IB, Chesters AK, J Fluid Mech, 329, 137 (1996). 